\title{
Baixo peso ao nascer em coorte de recém-nascidos em Goiânia-Brasil no ano de 2000
}

\author{
Low birth weight in a cohort of newborns in Goiânia-Brazil in 2000
}

Margareth Rocha Peixoto Giglio ${ }^{1}$, Joel Alves Lamounier ${ }^{2}$, Otaliba Libânio de Morais Neto ${ }^{3}$, Cibele Comini César ${ }^{4}$

\section{RESUMO}

\begin{abstract}
Objetivo: analisar o peso ao nascer da coorte de recém-nascidos do ano 2000, em Goiânia, pela determinação do coeficiente de mortalidade e probabilidade de sobrevivência neonatal, estratificados por categorias de peso ao nascer e, ainda, pela identificação dos fatores associados ao baixo peso ao nascer (BPN). Métodos: estudo de coorte retrospectivo, constituído por linkage dos arquivos do SIM (Sistema de Informações de Mortalidade) e do SINASC (Sistema de Informações de Nascimentos). Foram calculados coeficientes de mortalidade neonatal para as categorias de peso ao nascer e construído um gráfico de probabilidades de sobrevivência neonatal por meio de análise de regressão linear. Foram identificados fatores de risco para o BPN mediante análise univariada (RR) e regressão logística, considerando-se nível de significância de 5\%. Resultados: a incidência de BPN foi de 6,9\%, sendo que $140(66,8 \%)$ óbitos neonatais ocorreram nesse grupo. Trinta por cento dos óbitos se deram na categoria de peso entre 1.500-2.500 g. Os fatores identificados como de risco para o BPN foram: prematuridade, presença de malformações congênitas, mães com idade em extremos reprodutivos, residência na região noroeste do município, baixo número de consultas no pré-natal, parto em hospital público e sexo feminino. Conclusão: a incidência de BPN foi semelhante aos países desenvolvidos e os coeficientes de mortalidade neonatal, por categoria de peso, aquém dos encontrados naqueles países. Os resultados encontrados orientam atenção para: prematuridade, hospitais públicos e região noroeste de Goiânia.
\end{abstract}

PALAVRAS-CHAVE: Mortalidade neonatal; Peso ao nascer; Baixo peso ao nascer; Prematuro; Desenvolvimento embrionário e fetal.

\section{ABSTRACT}

Purpose: to analyze birth weight in a cohort of newborns for the year 2000, in Goiânia, by determining the coefficient of mortality and neonatal survival probability, stratified by categories of birth weight, and also, through the identification of factors associated with low birth weight (LBW). Methods: a retrospective cohort study, made possible by the linkage of data from the ISM (Information System on Mortality) and ISLB (Information System on Live Births) files. Coefficients of neonatal mortality were calculated for the categories of birth weight and a neonatal survival probability chart was constructed with the help of linear regression analysis. Risk factors for LBW were identified by univariate analysis (RR) and logistic regression analysis, and the level of significance was set at 5\%. Results: the incidence of LBW was 6.9\% and $140(66.8 \%)$ neonatal deaths took place in this group. Thirty percent of these deaths occurred in the 1,500-2,500 g weight bracket. The following risk factors were identified for LBW: preterm pregnancy, presence of congenital malformations, mothers at the extreme ages for reproduction, mothers living in the northwestern region of the city, insufficient prenatal appointments with the doctor, delivery in a public hospital, and female babies. Conclusion: Goiânia had an incidence of LBW which is comparable to that of developed countries and coefficients of neonatal mortality by category of weight were below those found for those countries. These results recommend that we pay attention to: prematurity, public hospitals, and the northwestern region of Goiânia.

KEYWORDS: Infant mortality; Birth weight; Infant, low birth weight; Infant, premature; Embryo and fetal development; Survival rate

\footnotetext{
1 Pós-Graduanda da Faculdade de Medicina da Universidade Federal de Minas Gerais, UFMG (MG).

2 Professor Adjunto do Departamento de Pediatria da Faculdade de Medicina - UFMG (MG).

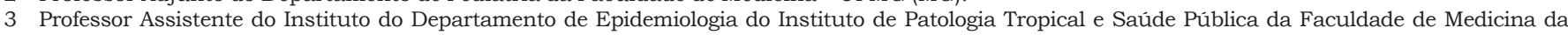
Universidade Federal de Goiás - UFGO (GO).

4 Departamento de Estatística do Instituto de Ciências Exatas - UFMG (MG).

Correspondência: Margareth Rocha Peixoto Giglio

Rua 9, 650, apto. 901 - setor Oeste - 74110-100 - Goiás - GO - e-mail: hgiglio@terra.com.br

Recebido em: 18/2/2005 Aceito com modificações em: 30/3/2005
} 
Introdução

Cerca de 7,1 milhões de crianças morrem a cada ano, em todo o mundo, no primeiro ano de vida. Metade dessas mortes ocorre no período neonatal sendo que, nesse período, $75 \%$ dos óbitos se dão na primeira semana e $40 \%$ nas primeiras 24 horas de vida. Aproximadamente $98 \%$ de todas as mortes neonatais ocorrem em países em desenvolvimento ${ }^{1}$.

São várias as causas de morte entre os recém-nascidos (RN), como: infecções, asfixia de parto, anomalias congênitas e complicações relacionadas à prematuridade. No entanto, o baixo peso ao nascer (BPN), ou seja, inferior a $2.500 \mathrm{~g}$, responde por uma parcela significante das mortes neonatais - cerca de 40 a $70 \%{ }^{1}$.

O peso ao nascer é, isoladamente, o principal fator associado ao risco de morte no período neona$\mathrm{tal}^{2}$. Complexo processo resultante de uma série de fatores de origem biológica, social e ambiental, o peso ao nascer é forte preditor da probabilidade de sobrevivência nos primeiros 28 dias de vida. Seu estudo coloca em evidência fatores relacionados à condição de vida, que podem estar modificando o potencial genético para a distribuição do peso ao nascer em determinada região e, dessa forma, estar influenciando sobre a mortalidade infantil ${ }^{3-5}$.

Os coeficientes de mortalidade neonatal são amplamente usados como indicadores sensiveis da qualidade de assistência à gestação, parto e ao RN de uma dada população. Por outro lado, a probabilidade de sobrevivência neonatal, estratificada por grupos de peso ao nascer, permite formular prognósticos e orientar condutas obstétricas antenatais diante da necessidade de se interromper uma gestação de alto risco ${ }^{6-8}$.

A cidade de Goiânia apresenta uma população de 1.093.007 habitantes, dos quais 105.100 são crianças menores de cinco anos de idade com coeficientes de mortalidade infantil e neonatal de, respectivamente, 16/1000 e 11/1000 habitantes ${ }^{9}$ e incidência de BPN de $6,8 \%{ }^{10}$. A grande parcela do componente neonatal para o coeficiente de mortalidade infantil coloca esse período como prioridade das politicas de saúde quando se pretende reduzir o coeficiente de mortalidade infantil nesse município, e o estudo do peso ao nascer funciona como estratégia para atingir esse alvo.

O objetivo do presente estudo é analisar o peso ao nascer da coorte de RN de Goiânia, no ano 2000, pela determinação do coeficiente de mortalidade e probabilidade de sobrevivência neonatal, estratificados por categorias de peso ao nascer e, ainda, pela identificação dos fatores associados ao
BPN entre as variáveis epidemiológicas e demográficas, referidas na Declaração de Nascimento (DN) do Sistema Nacional de Nascidos Vivos (SINASC).

\section{Métodos}

Estudo de coorte retrospectivo obtido a partir de linkage entre as DN do SINASC e a Declaração de Óbito (DO) do Sistema de Informações de Morte (SIM), fornecidas pelas Secretarias Municipal e Estadual de Saúde. Os óbitos registrados em DO foram investigados pelo grupo de monitoramento dos óbitos neonatais, no municipio de Goiânia, constituído por um epidemiologista, dois pediatras, uma obstetra, uma enfermeira e uma psicóloga. Essa investigação foi realizada por meio de busca em prontuários no hospital de ocorrência do óbito, no hospital de ocorrência do parto, nas fichas de consultas pré-natais e mediante visitas domiciliares em que as mães foram entrevistadas. Reavaliou-se, assim, a causa básica dos óbitos neonatais de acordo com o Código Internacional de Doenças (CID 10).

Entre 21 variáveis contidas na DN, que poderiam fornecer informações para estudo, seis não foram incluídas por apresentarem mais de $10 \%$ de perdas de informação. A única exceção se deve à inclusão da variável "escolaridade materna" que, apesar de apresentar indice de perdas de 10,4\%, foi incluída devido à sua importância como indicador socioeconômico e, também, porque a distribuição de algumas variáveis mostrou-se heterogênea nos grupos com e sem informação para a escolaridade materna. A ocorrência do óbito e a idade do RN ao óbito foram as únicas variáveis da DO consideradas para estudo, seguindo orientação de estudos prévios que mostraram baixa qualidade do seu preenchimento ${ }^{11,12}$.

Foram incluídos na categoria BPN os RN com peso inferior a $2.500 \mathrm{~g}$, como período neonatal os primeiros 28 dias de vida e como pré-termo a idade gestacional ao nascer inferior a 37 semanas $^{13}$. Definiu-se como variável dependente o BPN e como variáveis de exposição: idade, escolaridade e região de residência da mãe, número de consultas no pré-natal, categoria do hospital de nascimento, idade gestacional, sexo e presença de malformações no RN.

Os hospitais foram agrupados em três categorias: público, privado sem atendimento ao SUS e privado com atendimento ao SUS, segundo o cadastro de estabelecimentos hospitalares da Secretaria de Saúde do Estado de Goiás e o cadastro de hospitais do Sistema de Informações Hospitalares 
do Ministério de Saúde (SIH). Na categoria hospital público, foram agrupados: três maternidades públicas estaduais, uma maternidade pública municipal, a maternidade-escola da Faculdade de Medicina da Universidade Federal de Goiás e um hospital filantrópico. Apesar de o hospital filantrópico estar referido na categoria de hospital privado com atendimento ao SUS pelo cadastro do SIH, esse hospital foi incluído na categoria de hospital público por apresentar fluxo de pacientes e atendimento semelhante a esses, apresentando inclusive serviços de residência médica e de estágios de profissionais da área médica. A categoria hospital privado com atendimento ao SUS foi constituída por 16 hospitais e a de hospital privado sem atendimento ao SUS por 6 hospitais.

Os bairros de residência da mãe foram agrupados em onze regiões ou distritos sanitários, de acordo com Instituto de Planejamento da Prefeitura Municipal de Goiânia (IPLAN) e, após a análise prévia que mostrou mortalidade neonatal bem maior na região noroeste em relação às demais regiões, a região de residência da mãe foi agrupada em duas categorias: região noroeste e outras regiões.

Foram incluídos no estudo todos os RN vivos, de mães residentes no município de Goiânia, durante o período de janeiro a dezembro do ano de 2000, e todos os óbitos neonatais, referentes a essa coorte. A Figura 1 mostra a formação do banco de dados, os casos incluídos e os excluídos.

Foram calculados coeficientes de mortalidade neonatal para as categorias de peso ao nascer, construídas com intervalos de $250 \mathrm{~g}$. O cálculo foi feito em relação a mil habitantes, utilizando-se como numerador o número de óbitos ocorridos naquela categoria e como denominador o número de RN com peso dentro de cada categoria.

Construiu-se um gráfico de probabilidade de sobrevivência no período neonatal, por categoria de peso ao nascer, utilizando-se a análise de regressão linear, com definição dos respectivos intervalos de confiança a 95\% e validação estatística dada pelo teste $\mathrm{F}$ com nivel de significância de $5 \%$.

As associações entre o BPN e as variáveis de exposição foram estudadas por meio de análise univariada, com o cálculo do risco relativo (RR), e por análise de regressão logística. Nos dois casos foram estabelecidos intervalos de confiança a 95\% com nivel de significância de $5 \%$. A validação estatística foi dada pelo teste $\chi^{2}$, com nivel de significância de $5 \%$, na análise univariada, e pelo teste de Wald na análise de regressão logística.

Considerando-se que variáveis com riscos relativos de grande magnitude podem mascarar $o$ efeito de outras, foram considerados dois modelos para a análise de regressão logística. No primeiro modelo, foram analisadas todas as variáveis de exposição em conjunto, no segundo foram excluídas as variáveis: idade gestacional e presença de malformação congênita. O objetivo desse procedimento foi analisar o impacto que essas duas variáveis, fortemente associadas ao BPN, poderiam ter no comportamento das outras.

Para a construção do banco de dados, análise e validação estatística das associações encontradas foram utilizados os programas de informática FoxPro 5.0, Epi-Info 6.04 e SPSS 11.5, e para a organização das referências bibliográficas foi utilizado o programa EndNote 2.2.

O presente estudo foi aprovado pelo Comitê de Ética e Pesquisa da Faculdade de Medicina da Universidade Federal de Goiás e, ainda, pelo Comitê de Ética e Pesquisa da Universidade Federal de Minas Gerais.

\section{Resultados}

A análise da coorte mostrou que foram registrados em Goiânia, durante o ano 2000, 19.653 nascimentos (Figura 1), sendo que 1124 $(6,9 \%)$ desses com diagnóstico de BPN. Ocorreram 206 óbitos neonatais, ressaltando-se que cerca de $140(71 \%)$ ocorreram no grupo com peso inferior a 2.500 g. Os coeficientes de mortalidade infantil e neonatal foram, respectivamente, 15,8/1000 e 10,5/1000 nascidos vivos.

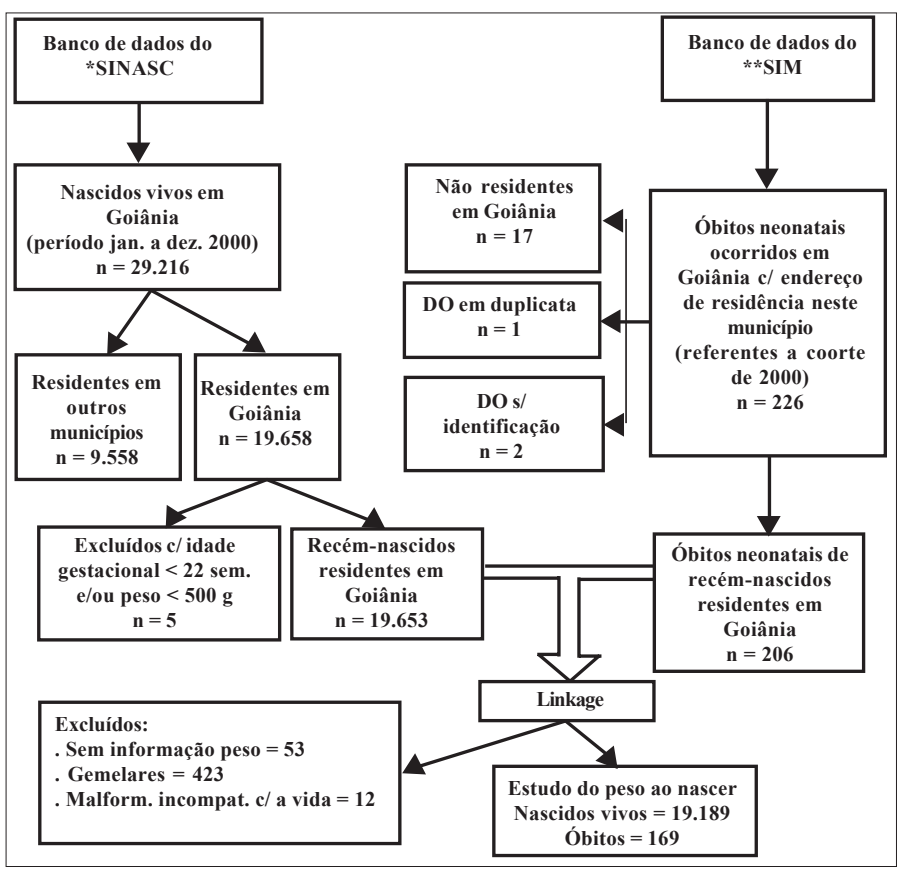

*SINASC: Sistema de Informação de Nascidos Vivos (Ministério da Saúde) **SIM: Sistema de Informação de Mortalidade (Ministério da Saúde) DO = Declaração de óbito

Figura 1 - Formação do banco de dados do estudo do peso ao nascer em Goiânia no ano de 2000. 
Os coeficientes de mortalidade neonatal, especificados por categorias de peso ao nascer (Tabela 1), evidenciaram a inversa relação entre as duas variáveis, ou seja, quanto menor o peso ao nascer maior foi o coeficiente de mortalidade.

Tabela 1 - Coeficientes de mortalidade neonatal por categorias de peso ao nascer em Goiânia, no ano de 2000.

\begin{tabular}{|c|c|c|c|}
\hline \multirow{2}{*}{$\begin{array}{c}\text { Peso ao nascer } \\
\text { em gramas }\end{array}$} & \multicolumn{2}{|c|}{ Óbitos } & \multirow{2}{*}{$\begin{array}{c}\text { Coeficiente mortalidade } \\
\text { Neonatal/1000 }\end{array}$} \\
\hline & $\mathrm{n}$ & $\%$ & \\
\hline $500-750$ & 16 & 80,0 & 800 \\
\hline $750-999$ & 10 & 62,5 & 625 \\
\hline $1.000-1.249$ & 26 & 56,5 & 565 \\
\hline $1.250-1.499$ & 18 & 34,6 & 346 \\
\hline $1.500-1.749$ & 14 & 20,0 & 200 \\
\hline $1.750-1.999$ & 9 & 6,3 & 63 \\
\hline $2.000-2.249$ & 12 & 4,7 & 47 \\
\hline $2.250-2.499$ & 8 & 1,5 & 15 \\
\hline $2.500-3.999$ & 56 & 0,3 & 3 \\
\hline$\geq 4.000$ & 5 & 0,5 & 5 \\
\hline
\end{tabular}

A Figura 2 mostra as probabilidades de sobrevivência ao período neonatal de acordo com o peso ao nascer e os respectivos intervalos de confiança $(95 \%)$ e nivel de significância (5\%). Nota-se que os intervalos de confiança são maiores nas extremidades da curva.

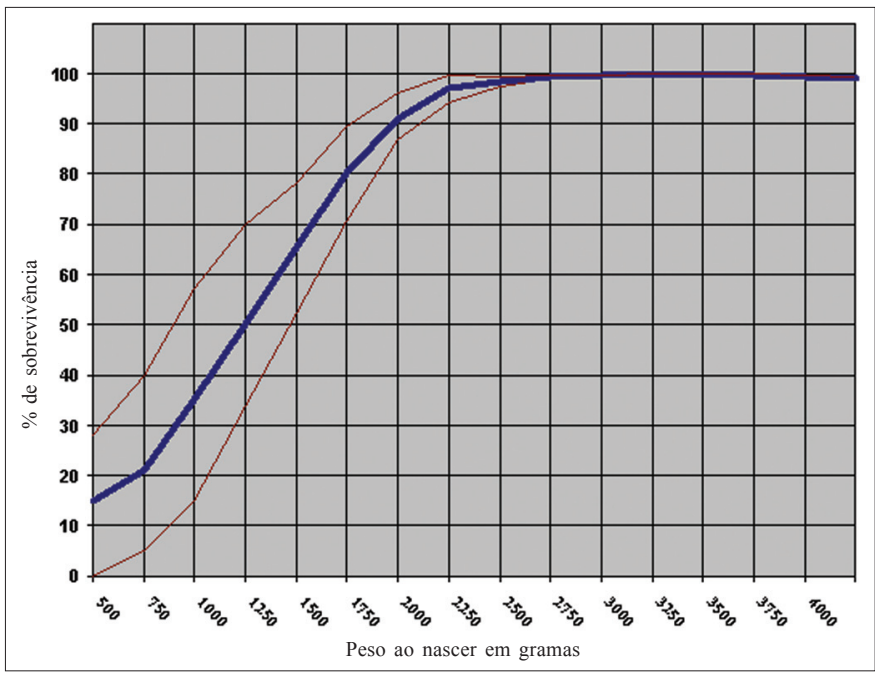

Figura 2 - Gráfico de probabilidades de sobrevivência neonatal por peso ao nascer, em Goiânia - 2000.

A Tabela 2 mostra a força da associação entre o BPN e as variáveis de exposição que foram significativas nos dois modelos considerados para estudo. Apesar de todas as variáveis testadas apre- sentarem associação com BPN na análise univariada, a variável escolaridade materna não apresentou associação estatisticamente significativa na análise de regressão logística. No primeiro modelo considerado para análise de regressão, considerando-se todas as variáveis, as variáveis idade gestacional, malformação congênita, categoria de hospital de nascimento, região de residência da mãe e sexo apresentaram-se estatisticamente associadas ao BPN. No segundo modelo, que excluiu as variáveis: idade gestacional e malformação congênita, além das variáveis estatisticamente significativas no primeiro modelo, as variáveis consulta pré-natal e idade materna também se tornaram estatisticamente associadas ao BPN.

Tabela 2 - Fatores de risco para o baixo peso ao nascer em Goiânia, no ano de 2000 (análise de regressão logística)

\begin{tabular}{|c|c|c|c|c|}
\hline \multirow[t]{3}{*}{ Variá } & Peso ao & nascer & \multirow[t]{3}{*}{ OR } & \multirow[t]{3}{*}{ IC $95 \%$} \\
\hline & $<2500 \mathrm{~g}$ & $>=2500 \mathrm{~g}$ & & \\
\hline & n $\%$ & n $\quad \%$ & & \\
\hline
\end{tabular}

Idade gestacional a

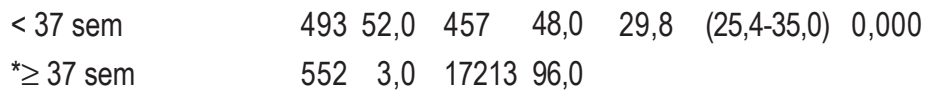

Malform. congênita $b$

Presente

*Ausente

$20 \quad 34,0 \quad 39 \quad 66,0$

$5,8 \quad(2,79-12,05) \quad 0,000$

Consultas no pré-natal c

Nenhuma

1 a 3

4 a 6

${ }^{*} \geq 7$

$926 \quad 5,4 \quad 16355 \quad 94,6$

Hospital do parto d

Público

Privado sem SUS

*Privado com SUS

$23 \quad 12,4 \quad 162 \quad 87,6$

$1,76(1,12-2,79) \quad 0,016$

$94 \quad 12,2 \quad 675 \quad 87,8 \quad 1,93 \quad(1,51-2,47) \quad 0,000$

$\begin{array}{llllllll}289 & 8,7 & 3037 & 91,3 & 1,5 & (1,28-1,75) & 0,000\end{array}$

$\begin{array}{llll}611 & 4,7 & 12446 \quad 95,3\end{array}$

Região residência e

Noroeste

*Outras regiões

$\begin{array}{llll}190 & 7,9 & 2209 & 92,1\end{array}$

$1,41 \quad(1,19-1,67) \quad 0,000$

Idade materna $f$

$$
\begin{aligned}
& <20 \text { anos } \\
& { }^{*} 20-34 \text { anos } \\
& \geq 35 \text { anos }
\end{aligned}
$$

$923 \quad 5,5 \quad 15718 \quad 94,5$

$2,76 \quad(2,28-3,2) \quad 0,000$ $222 \quad 4,9 \quad 4233 \quad 95,1 \quad 1,35 \quad(1,12-1,60) \quad 0,001$ $\begin{array}{llll}483 & 4,3 & 1091 & 95,7\end{array}$

Sexo $g$

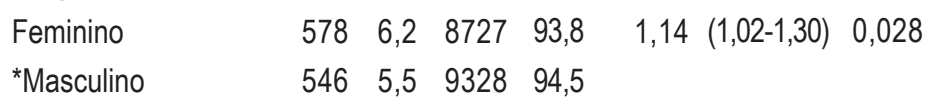

*Categorias de referência para cálculo do O.R.

Excluídos: a 474 (2,5\%) s/ informação idade gestacional, b 1849 (9,6\%) s/ informação malformação congênita, c $1852(9,6 \%)$ s/ informação consultas no pré-natal, d 28 (0,14\%) s/ informação local do parto, e $149(0,78 \%)$ s/ informação região residência, f $203(1,0 \%)$ s/ informação idade materna e g $10(0,052 \%)$ s/ informação sexo. 


\section{Discussão}

A incidência de BPN de 6,9\%, encontrada neste estudo, foi menor que a média nacional, e a ocorrência de BPN apresentou íntima associação com fatores descritos na literatura como de risco para a mortalidade neonatal ${ }^{3,4,11}$.

A estimativa corrigida de BPN para o país, em 1996 , foi de $8,5 \%$, com variações de $7,8 \%$ (região Sul) a 9,4\% (região Centro-Oeste), podendo os dados das regiões Norte e Nordeste estarem subestimados devido aos altos índices de subnotificação. A média mundial para o BPN em países desenvolvidos tem sido de $6 \%$ e de $18 \%$ para os países subdesenvolvidos ${ }^{5}$.

A maior contribuição desse estudo foi propiciar comparações das chances de sobrevivência neonatal e da distribuição dos óbitos neonatais, por categorias de peso, entre Goiânia e outras regiões. Pode-se concluir que, por um lado, esse município pode ser considerado privilegiado no ranking da mortalidade infantil e incidência de BPN, se comparado a outros estados do Brasil e vários países menos desenvolvidos ${ }^{5,13}$. Por outro lado, apresenta probabilidades de sobrevivência neonatal, por categorias de peso ao nascer, bem menores em relação à dos países desenvolvidos, para RN na mesma categoria $^{7,8}$. Nesse município, um RN com peso aproximado de $1.000 \mathrm{~g}$ apresentou $35 \%$ de chance de sobrevivência ao período neonatal. No Canadá, Estados Unidos e vários países da Europa a chance seria duas vezes maior ${ }^{6-8}$.

Comparando-se o coeficiente de mortalidade neonatal, distribuição dos óbitos infantis e incidência de BPN com os descritos por Morais Neto e Barros $^{10}$, para esse mesmo municipio em 1992, observa-se que esses, praticamente, não mudaram nos últimos oito anos. A mortalidade neonatal encontrada por esses autores foi de 11/1000 nascidos vivos, cerca de $67,5 \%$ das mortes no primeiro ano de vida também ocorreram no período neonatal e a incidência de BPN foi de $6,8 \%$, sendo que $64,2 \%$ dos óbitos ocorreram nesse grupo de peso.

A estratificação do coeficiente de mortalidade neonatal, por peso ao nascer, mostrou que $30 \%$ dos óbitos neonatais ocorreram na faixa de peso entre $1.500-2.500$ g e $26 \%$ na faixa superior a 2.500 g. Nos países desenvolvidos, a mortalidade neonatal se dá, principalmente, no grupo de RN prematuros com muito baixo peso ao nascer (MBPN), ou seja, inferior a $1.500 \mathrm{~g}^{6-8}$. Nesses países, o MBPN decorre de doenças, na gravidez, de dificil controle, como o descolamento prematuro da placenta, a doença hipertensiva da gra- videz e a placenta prévia ${ }^{2,14}$. No nosso meio, sabese que grande parte dos RN de BPN (cerca de metade) são pequenos para a idade gestacional (PIG), não necessariamente prematuros, decorrentes de fatores ambientais e relacionados aos aspectos socioeconômicos ${ }^{4,5}$. Nesse estudo a prematuridade foi responsável por 493 (47,2\%) casos de BPN. A ocorrência do PIG e a correlação entre as categorias de idade gestacional e do peso ao nascer não puderam ser avaliadas uma vez que a variável "idade gestacional" é referida na DN de forma categorizada. Estudos descritos na literatura que utilizaram, na análise de sobrevida neonatal, a associação da idade gestacional e peso ao nascer, mostraram maior precisão na predição das chances de sobrevivência após o nascimento ${ }^{6-8}$. Lansky et al. ${ }^{11}$, em estudo que utiliza a classificação de Wigglesworth, avaliando as causas de mortes perinatais em Belo Horizonte em 1999, enfatizam que os óbitos perinatais que ocorrem em RN com peso superior a $1.500 \mathrm{~g}$, por imaturidade são decorrentes de má qualidade da assistência ao RN em sala de parto e berçário. Os óbitos por asfixia que ocorrem em RN com peso superior a $1.500 \mathrm{~g}$ decorrem de má assistência ao parto e ao RN na sala de parto e berçário e, ainda, da organização da rede assistencial para gestante e RN. Altas taxas de malformações fetais estão relacionadas às falhas no rastreamento e terapêutica prénatal de lesões potencialmente tratáveis ${ }^{11}$.

Os resultados dessa avaliação mostram padrão de distribuição da mortalidade neonatal, por categorias de peso, similar ao de vários estudos em todo o mundo. Os mais baixos coeficientes de mortalidade neonatal ocorreram no grupo de RN com peso entre 3.000-3.999 g e os mais altos no grupo com peso abaixo que $2.500 \mathrm{~g}$. No grupo com peso entre 2.500 e $3.000 \mathrm{~g}$, a chance de morrer no período neonatal foi duas vezes maior que no primeiro grupo. O grupo com peso superior a $4.000 \mathrm{~g}$ apresentou mortalidade neonatal mais elevada que o grupo com peso entre 3.000 e 3.999 g; no entanto, a quantidade de nascimentos, nesse grupo, foi inexpressiva. A mortalidade neonatal foi 32,43 vezes mais alta no grupo com $\mathrm{BPN}$ em relação ao grupo com peso igual ou maior que $2.500 \mathrm{~g}^{4}$.

O gráfico de probabilidades de sobrevivência neonatal, por peso ao nascer, mostra intervalos de confiança maiores nas extremidades da curva. Isso se deve à não homogeneidade na distribuição do peso ao nascer na população quando se verificou maior concentração de casos nos valores de peso entre 2.500 a $3.999 \mathrm{~g}(88,9 \%)$. Quanto menor o tamanho da amostra maior foi o intervalo de 
confiança. Amostras maiores, principalmente nas categorias menores de peso, são necessárias para predição mais fidedigna das probabilidades de sobrevivência neonatal.

Das informações analisadas, pode-se concluir que a ocorrência de BPN foi 29,8 vezes maior entre os RN prematuros em relação aos RN de termo 5,8 vezes maior nos RN portadores de malformações compativeis com a vida em relação aos não portadores, 2,7 vezes maior nos hospitais públicos em relação aos hospitais privados com atendimento ao SUS, 41\% maior na região noroeste em relação às demais regiões de Goiânia, 37 e $31 \%$ maior entre as mães adolescentes e com idade superior a 35 anos, respectivamente, em relação à mãe com idade entre 20 e 34 anos e $14 \%$ maior nos RN do sexo feminino em relação ao masculino. Esses resultados são semelhantes aos que têm sido descritos na literatura ${ }^{3-5}$.

$\mathrm{Na}$ análise univariada, verificou-se relação inversa entre o BPN e o número de consultas durante o pré-natal e grau de instrução da mãe. Quanto maior o número de consultas pré-natal e maior o grau de instrução da mãe, menor a ocorrência do BPN, resultados coerentes com os encontrados na literatura ${ }^{4,5}$. No entanto, a associação entre o grau de instrução materna e o BPN não foi confirmada por nenhum dos dois modelos considerados para análise de regressão logística,

Apesar de ter sido observada relação inversa entre o número de consultas no pré-natal e a ocorrência do BPN, 60,1\% dos RN com BPN eram de gestantes que tiveram 7 consultas ou mais; $75 \%$ das mães tiveram sete ou mais consultas e apenas $1 \%$ não foi a nenhuma consulta. A maioria das gestantes compareceu a consultas em número superior ao preconizado pelo Ministério de Saúde. Isso chama a atenção para a qualidade de assistência ao pré-natal, que pode estar sendo falha na identificação dos casos e na prevenção do $\mathrm{BPN}^{5}$.

A ocorrência do BPN foi maior nos extremos da vida reprodutiva. As mães adolescentes e as mães com idade igual ou superior a 35 anos tiveram, respectivamente, 37 e $31 \%$ mais $\mathrm{RN}$ com baixo peso em relação às mães com idade entre 20 e 34 anos, resultados estes, coerentes com os encontrados na literatura ${ }^{3,15}$. As razões para a maior incidência de BPN entre mães adolescentes não estão claramente estabelecidas na literatura. As razões mais freqüentemente citadas são a imaturidade física e a pior nutrição entre as adolescentes. Horon et al. ${ }^{15}$, comparando gestantes adolescentes e adultas na Pensilvânia, chamou a atenção para o fato de que as mães adolescentes são mais comumente primigrávidas, de baixo poder socioeconômico e oriundas de regiões mais carentes, fatores descritos na literatura como associados ao BPN e à mortalidade infantil. A associação de BPN com idade materna superior a 35 anos parece estar relacionada à maior incidência, nessa faixa etária, de intercorrências na gravidez, como diabetes e hipertensão arterial, que modificam o intercurso da gestação e aumentam a ocorrência de partos prematuros. Além disso, a incidência de malformações congênitas, decorrentes de cromossomopatias, é maior nessa faixa etária ${ }^{15}$.

Vários estudos têm mostrado a maior associação entre malformações congênitas, BPN e prematuridade $^{3,11,14-16}$. Não é tarefa fácil controlar a incidência de malformações congênitas em uma população. No entanto, alguns casos de óbitos neonatais estiveram associados a malformações decorrentes de doenças infecciosas, ocorridas durante a gravidez, passiveis de prevenção, como a rubéola, e de tratamento, como a sífilis e a toxoplasmose $^{16}$.

Quando se parte de índices de BPN relativamente baixos, como o encontrado nesse estudo, a prevenção do BPN pode ser tarefa dificil ${ }^{5}$. Uma intervenção promissora poderia ser a diminuição da incidência da prematuridade e, principalmente, da mortalidade decorrente dessa. Nesse sentido, maior atenção deve ser direcionada à prematuridade por meio da identificação dos seus principais fatores de risco durante o pré-natal, da utilização de corticoterapia naqueles casos de alto risco para o parto prematuro e no encaminhamento dos casos de parto prematuro para serviços terciários, nos quais a assistência ao parto e ao RN é mais adequada $^{5,15}$.

A maior incidência do BPN na região noroeste de Goiânia e nos hospitais públicos, encontrada nesse estudo, exemplificam a influência de fatores socioeconômicos na distribuição do peso ao nascer. Essa região, bem definida geograficamente, caracteriza-se pela concentração de uma população de baixo poder socioeconômico, maior proporção de mães adolescentes, menor nivel de escolaridade materna e maiores coeficientes de mortalidade infantil do municipio ${ }^{10}$. A maior ocorrência de BPN nessa região, associado a outros indicadores de saúde, deve orientar as ações de saúde e as políticas públicas para essa área do municipio.

Por outro lado, os hospitais públicos, locais que dão assistência perinatal a maioria da população de risco para o BPN, devem ser priorizados com melhorias na qualidade de assistência à gestante e ao RN através da capacitação de pessoal médico e paramédico e, ainda, da disponibilidade 
de tecnologia avançada em unidades de cuidados intensivos neonatais.

Os resultados encontrados nesse estudo mostraram coerência com os de outros estudos realizados no Brasil e em outros países. A utilização da vinculação dos bancos de dados do SINASC e do SIM permitiu a identificação de fatores associados à ocorrência do BPN em Goiânia. Mostrase, assim, que é viável a utilização desses sistemas para estudos de coorte populacionais. A utilização crítica das informações contidas nesses dois instrumentos, DN e DO, pode fornecer subsídios para implementação de estratégias de redução da mortalidade infantil, para definir critérios a serem utilizados no monitoramento de $\mathrm{RN}$ de risco e, ainda, como fonte de indicadores para acompanhamento e avaliação de ações dirigidas à população materno-infantil.

Os resultados aqui encontrados poderão orientar os órgãos públicos de saúde no planejamento de ações destinadas à diminuição da mortalidade neonatal, especificamente para Goiânia. Por outro lado, esses resultados deverão auxiliar os profissionais de saúde a fazerem prognósticos das chances de sobrevida neonatal, também específicas para esse município, diante da necessidade de se interromper uma gestação de alto risco e, ainda, diante da necessidade de orientação à família quanto ao risco de morte do RN.

\section{Referências}

1. World Health Organization. Newborn health and survival: a call to action. In: Family and Community Health. Geneva: WHO; 2001.

2. World Health Organization. The incidence of lowbirth-weight: a critical review of available information. World Health Stat Q. 1980;33(3):197-224.

3. Costa CE, Gotlieb SLD. Estudo epidemiológico do peso ao nascer a partir da Declaração de Nascido Vivo. Rev Saúde Publica. 1998;32(4):328-34.

4. Patterns of birthweighs: a summary. Bull Pan Am Health Organ. 1987;21(2):185-95.

5. Victora CG. Intervenções para reduzir a mortalidade infantil pré-escolar e materna no Brasil. Rev Saúde Pública. 2001;4(1):3-69.
6. Cooper RL, Goldenberg RL, Creasy RK, DuBard MB, Davis RO, Entman SS, et al. A multicenter study of preterm birth and gestacional age-specific neonatal mortality. Am J Obstet Gynecol. 1993;168(1 Pt 1):78-84.

7. Draper ES, Manktelow B, Field DJ, James D. Prediction of survival for preterm births by weight and gestational age: retrospective population based study. BMJ. 1999;319(7217):1093-7.

8. Wen SW, Kramer MS, Liu S, Dzakpasu S, Sauve R. Infant mortality by gestational age and birth weight in Canadian provinces and territories, 1990-1994 births. Chronic Dis Can. 2000;21(1):14-22.

9. Instituto Brasileiro de Geografia e Estatística. IBGE. Estatísticas do registro civil. 2000 [citado 2003 jun 14]. Disponivel em: http://www.ibge.gov.br/ estatistica/populacao/censo2000

10. Morais Neto OL, Barros MBA. Fatores de risco para mortalidade neonatal e pós-neonatal na Região Centro-Oeste do Brasil: linkage entre bancos de dados de nascidos vivos e óbitos infantis. Cad Saúde Pública. 2000;16(2):477-85.

11.Lanski S, França E, Leal MC. Mortes perinatais evitáveis em Belo Horizonte, Minas Gerais, Brasil, 1999. Cad Saúde Pública. 2002;18(5):1389-400.

12.Paes NA, Albuquerque MEE. Avaliação da qualidade dos dados populacionais e cobertura dos registros de óbitos para as regiões brasileiras. Rev Saúde Pública. 1999;33(1):33-43.

13.World Health Organization. Perinatal mortality: a listing of available information. Geneva: Division of Family and Reproductive Health; 1996.

14.Berkowitz GS, Papiernik E. Epidemiology of preterm birth. Epidemiol Rev. 1993;15(2):414-43.

15. Horon IL, Strobino DM, MacDonald HM. Birth weights among infants born to adolescent and adult woman. Am J Obstet Gynecol. 1983;146(4):444-9.

16.Rassi Neto E, Morais Neto OL, Rosso CFW, Siqueira Junior JB, Afonso ET, Marques SM, et al. Monitoramento dos óbitos neonatais ocorridos no município de Goiânia-GO, 1999/2000. Painel apresentado no $1^{\circ}$ Congresso Mineiro de Epidemiologia e Saúde Pública; 2000 maio 24-27; Belo Horizonte, Brasil. 